\title{
Unresolved challenges in the microanalysis of actinides and nuclear materials
}

\author{
Philipp Pöml
}

European Commission - Joint Research Centre, United States

Quantitative X-ray microanalysis is a valuable tool for a wide range of nuclear applications. Typical examples for applications in the field of actinide materials are the analysis of fresh or spent nuclear fuel (post irradiation examination - PIE), the quantification of $\mathrm{Pu}$ or Am in materials used in radioisotope thermoelectric generators (RTGs) for space applications (see latest Mars rover Perseverance), or the analysis of materials formed during severe nuclear accidents like Three Mile Island, Chernobyl, and Fukushima [1-3].

The nuclear scientist is usually particularly interested in the actinides and their fission products or decay products, their quantities and spatial distribution in the materials of interest. The good news is, that in principle this is straight forward, because fission products are usually elements somewhere between bromine and terbium, hence rather "normal" elements, and for actinides starting with actinium there is in theory no upper limit of what can be analysed. Unfortunately, however, this is not so simple.

Many analysts will know the trouble the L-lines give, when analysing rare earth elements, for example monazites. All the lines are heavily overlapping and it is very difficult to do a proper background measurement and get all the overlap corrections right. For actinides this is just the same; here it is the Mlines. Fig. 1 shows an example WDS spectrum recorded using a PET crystal on a shielded Cameca SX100R electron probe microanalyzer (EPMA) operated at JRC Karlsruhe, Germany. The positions of the $\mathrm{Cm} \mathrm{M} \alpha$ and $\mathrm{M} \beta$ lines are only indicative, because no $\mathrm{Cm}$ containing material was available at that time. Visible are only the major M-lines. The actinide M-lines heavily overlap each other with some of the worst overlaps being $U \mathrm{M} \beta$ on $\mathrm{Pu} \mathrm{M} \alpha$ and $\mathrm{Np} \mathrm{M} \beta$ on Am M $\alpha$. There are several options to improve this situation. Commonly for actinide analysis a quartz 10-11 crystal is used instead of PET, the quartz offering a slightly better resolution at the cost of some intensity. In addition, the Rowland circle of the spectrometer can be increased. A standard Cameca spectrometer has a Rowland circle of $160 \mathrm{~mm}$. The shielded SX100Rs recently sold have a Rowland circle of $180 \mathrm{~mm}$. The old MS46 spectrometers previously used at JRC Karlsruhe even had a $220 \mathrm{~mm}$ Rowland circle. On top of that, the MS46 even had Johansson geometry quartz crystals, but these do not seem to be available anymore.

The lack of reference materials is another long-standing issue [4]. Th and $U$ can be easily commercially purchased either as metal or oxide, but for actinium $(Z=89)$ and elements above $Z=92$ it becomes more difficult. For most organizations operating an EPMA for actinide research it is relatively simple to obtain $\mathrm{NpO}_{2}$ and $\mathrm{PuO}_{2}$ ceramics as reference materials, because their activity is not too high and they can be easily polished and stored. It is, however, not so simple to get chemically pure $\mathrm{PuO}_{2}$, because ${ }^{241} \mathrm{Pu}$ is slowly decaying to ${ }^{241} \mathrm{Am}$ and hence the amount of $\mathrm{Pu}$ in the reference material is slowly decreasing while Am is increasing. For Am and $\mathrm{Cm}$ it is even more complicated. These elements are highly radioactive and it is difficult to obtain large enough quantities to actually produce a polished reference material. Even if one was obtained, the radiation damage from the decay of Am and $\mathrm{Cm}$ will mechanically destroy the material in a short time. Because Am and $\mathrm{Cm}$ have rather short half-lives the composition of reference materials would change rather quickly. Quantitative x-ray microanalysis of Bk or above has not been reported yet. 
Another problem can be uncertainties on parameters needed for matrix correction algorithms for quantitative x-ray microanalysis. The mass absorption coefficients (MACs) for light elements in an actinide bearing material are an example [5]. Most MAC tabulations stop at $U(Z=92)$ and the available values for $Z>92$ are mostly extrapolations of existing MAC tabulations. Fig. 2 shows a comparison of available MAC values for $\mathrm{O} \mathrm{K} \alpha$ in actinides and the most recent values obtained in [5]. It can be seen that the scatter is rather large. Because X-ray absorption is the dominant correction for ultra-light elements like $\mathrm{O}$, the difference in these values will have a tremendous effect on the quantitative result.

The analysis of actinides and radioactive materials in general remains challenging. Only a few of the associated problems could be covered in this abstract. It is, however, good to see that new procedures are being developed (e.g. overlap correction), new data becomes available (e.g. MACs for light elements), and better software tools are being developed (e.g. PENEPMA). Employing the new tools will certainly significantly improve the quality of results in this field.

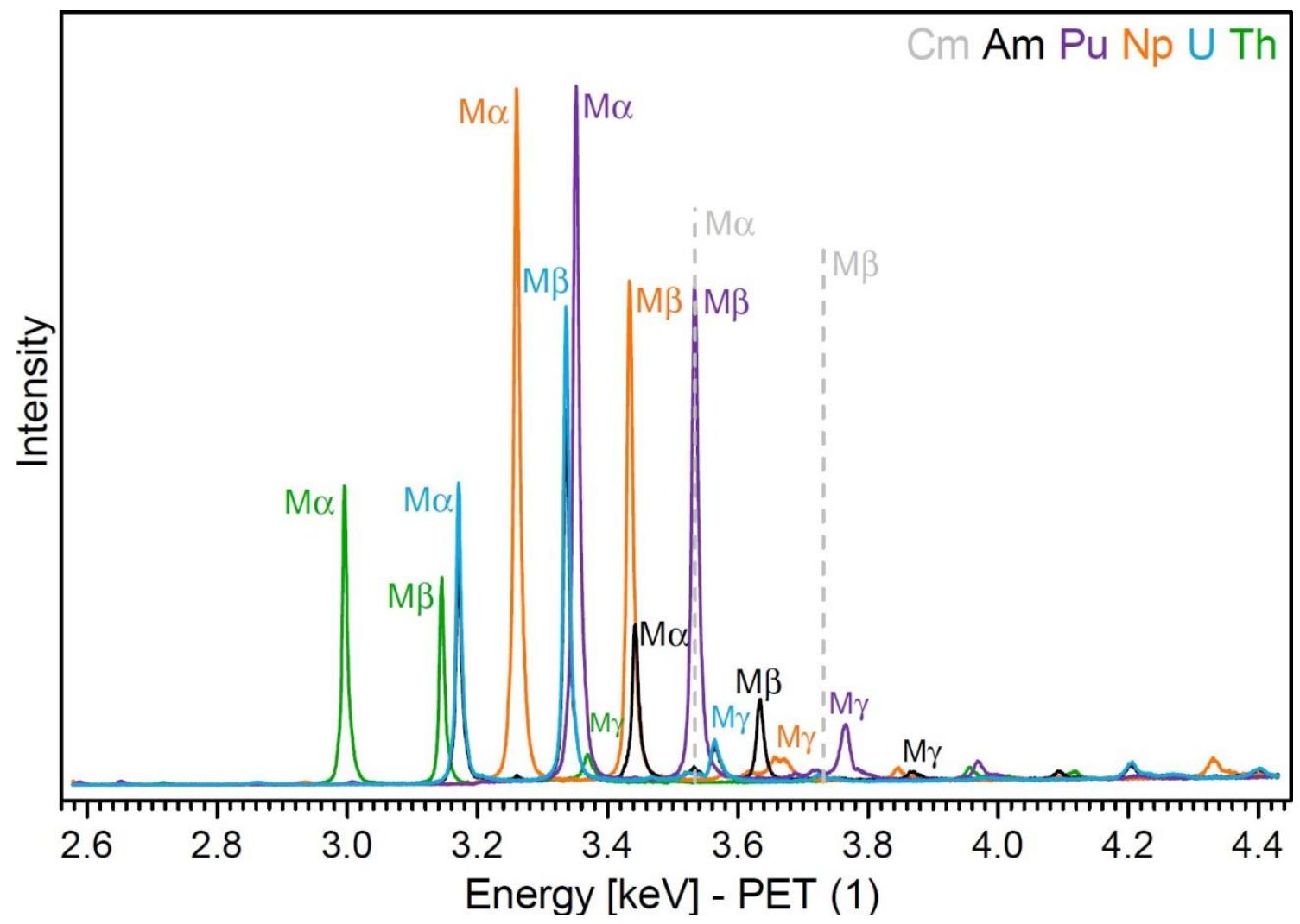

Figure 1. M-Line region for the actinides recorded using a PET crystal installed on a shielded Cameca SX100R EPMA. 


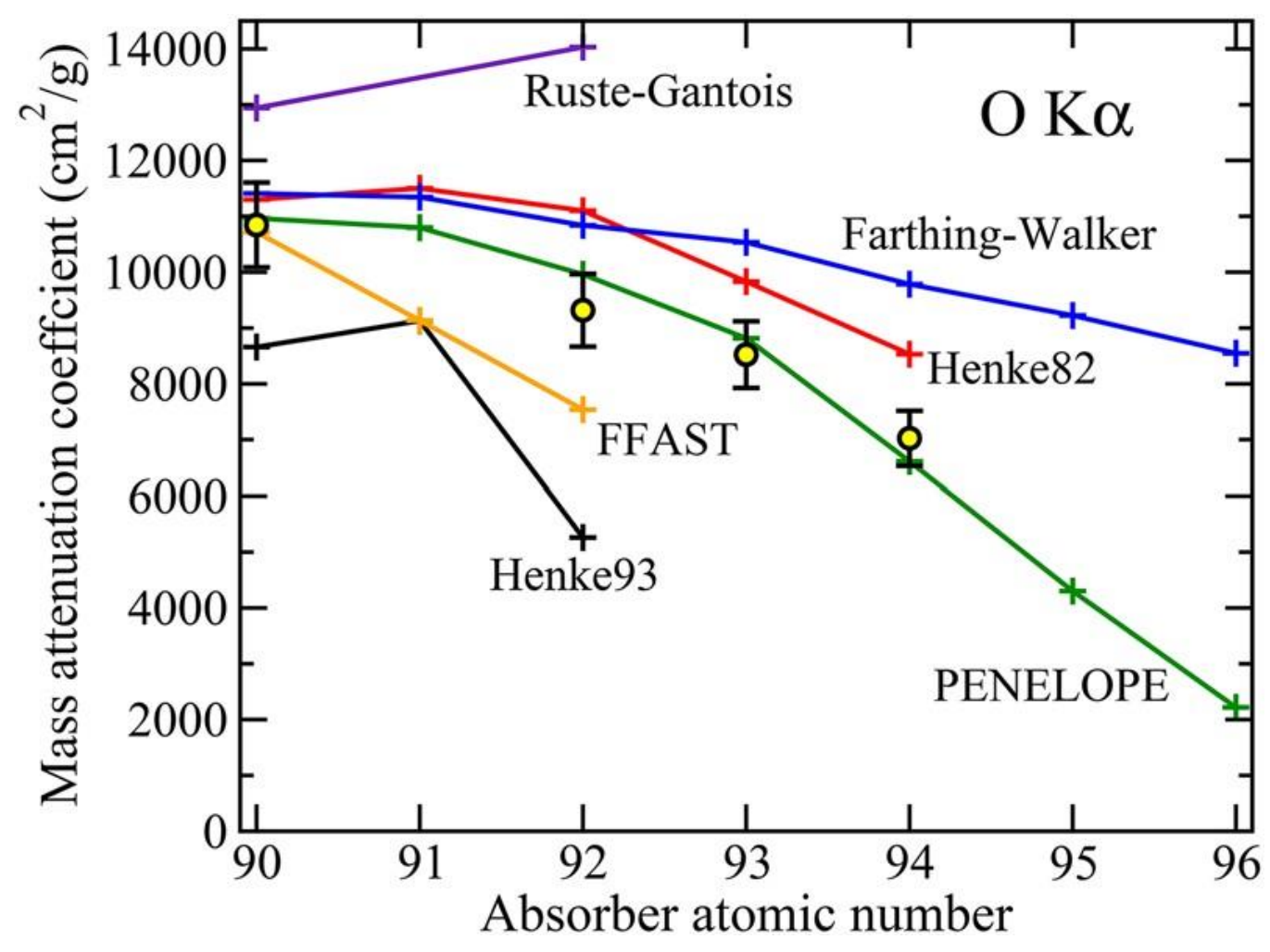

Figure 2. Comparison of tabulated MACs of actinide elements for $\mathrm{O} \mathrm{K} \alpha \mathrm{X}$-rays. Solid lines are MAC tabulations as indicated in the legends; open circles are experimental results from [5].

References

[1] C T Walker, et al., IOP Conf. Ser.: Mater. Sci. Eng., 32 (2012), p. 012028.

[2] J-F Vigier, et al., Inorg. Chem., 57 (2018), p. 4317-4327.

[3] P Pöml and B Burakov, J. Nucl. Mater., 488 (2017), p. 314-318.

[4] X Ritter, et al., Microsc. Microanal. (submitted).

[5] P Pöml and X Llovet, Microsc. Microanal., 26 (2020), p. 194-203. 